Chapman University

Chapman University Digital Commons

Physical Therapy Faculty Articles and Research

Physical Therapy

$12-2-2016$

\title{
Adaptations of Lumbar Biomechanics after Four Weeks of Running Training with Minimalist Footwear and Technique guidance: Implications for Running-Related Lower Back Pain
}

Szu-Ping Lee

University of Nevada, Las Vegas

Joshua P. Bailey

University of Nevada, Las Vegas

Jo Armour Smith

Chapman University, josmith@chapman.edu

Stephanie Barton

University of Nevada, Las Vegas

David Brown

University of Nevada, Las Vegas

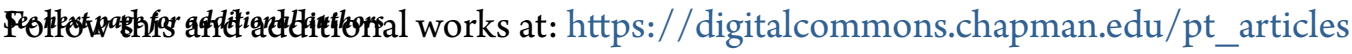

Part of the Kinesiotherapy Commons, $\underline{\text { Musculoskeletal System Commons, Physical Therapy }}$ Commons, and the Sports Sciences Commons

\section{Recommended Citation}

Lee S, Bailey JP, Smith JA, Barton S, Brown D, Joyce T. Adaptations of lumbar biomechanics after four weeks of running training with minimalist footwear and technique guidance: Implications for running-related lower back pain. Phys Ther Sport. 2018;29:101-107. doi: 10.1016/j.ptsp.2016.11.004 


\section{Adaptations of Lumbar Biomechanics after Four Weeks of Running Training with Minimalist Footwear and Technique guidance: Implications for Running-Related Lower Back Pain}

Comments

NOTICE: this is the author's version of a work that was accepted for publication in Physical Therapy in Sport. Changes resulting from the publishing process, such as peer review, editing, corrections, structural formatting, and other quality control mechanisms may not be reflected in this document. Changes may have been made to this work since it was submitted for publication. A definitive version was subsequently published in Physical Therapy in Sport, volume 29, in 2018. DOI: 10.1016/j.ptsp.2016.11.004

The Creative Commons license below applies only to this version of the article.

\section{Creative Commons License}

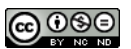

This work is licensed under a Creative Commons Attribution-Noncommercial-No Derivative Works 4.0 License.

\section{Copyright}

Elsevier

Authors

Szu-Ping Lee, Joshua P. Bailey, Jo Armour Smith, Stephanie Barton, David Brown, and Talia Joyce 


\title{
ADAPTATIONS OF LUMBAR BIOMECHANICS AFTER A FOUR-WEEK RUNNING \\ TRAINING WITH MINIMALIST FOOTWEAR AND TECHNIQUES: \\ IMPLICATIONS FOR RUNNING-RELATED LOWER BACK PAIN
}

\author{
Szu-Ping Lee, PT, $\mathrm{PhD}^{1}$ \\ Joshua P. Bailey, MS ${ }^{2}$ \\ Jo Armour Smith, PT, $\mathrm{PhD}^{3}$ \\ Stephanie Barton, DPT ${ }^{1}$ \\ David Brown, DPT ${ }^{1}$
}

Talia Joyce, DPT ${ }^{1}$

${ }^{1}$ Department of Physical Therapy, University of Nevada, Las Vegas, Nevada, USA

${ }^{2}$ Department of Kinesiology \& Nutritional Science, University of Nevada, Las Vegas, Nevada, USA

${ }^{3}$ Department of Physical Therapy, Chapman University, Orange, California, USA

Corresponding author:

Szu-Ping Lee, PT, PhD

Department of Physical Therapy, University of Nevada, Las Vegas

4505 S. Maryland Parkway, Box 453029, Las Vegas, NV 89154-3029, USA

Phone: (702)895-3086

Email: szu-ping.lee@unlv.edu 
- A 4-week minimalist style running training was conducted in recreational runners.

- After training, runners exhibited reduced lumbar extension angle during stance.

- After training, runners exhibited reduced lumbar paraspinal muscle activation.

- Changes in lumbar kinematics and muscle activation transferred to normal running.

- No runner reported any adverse effect during the 4-week training. 
*Manuscript including abstract and title but excluding Author information

Click here to view linked References

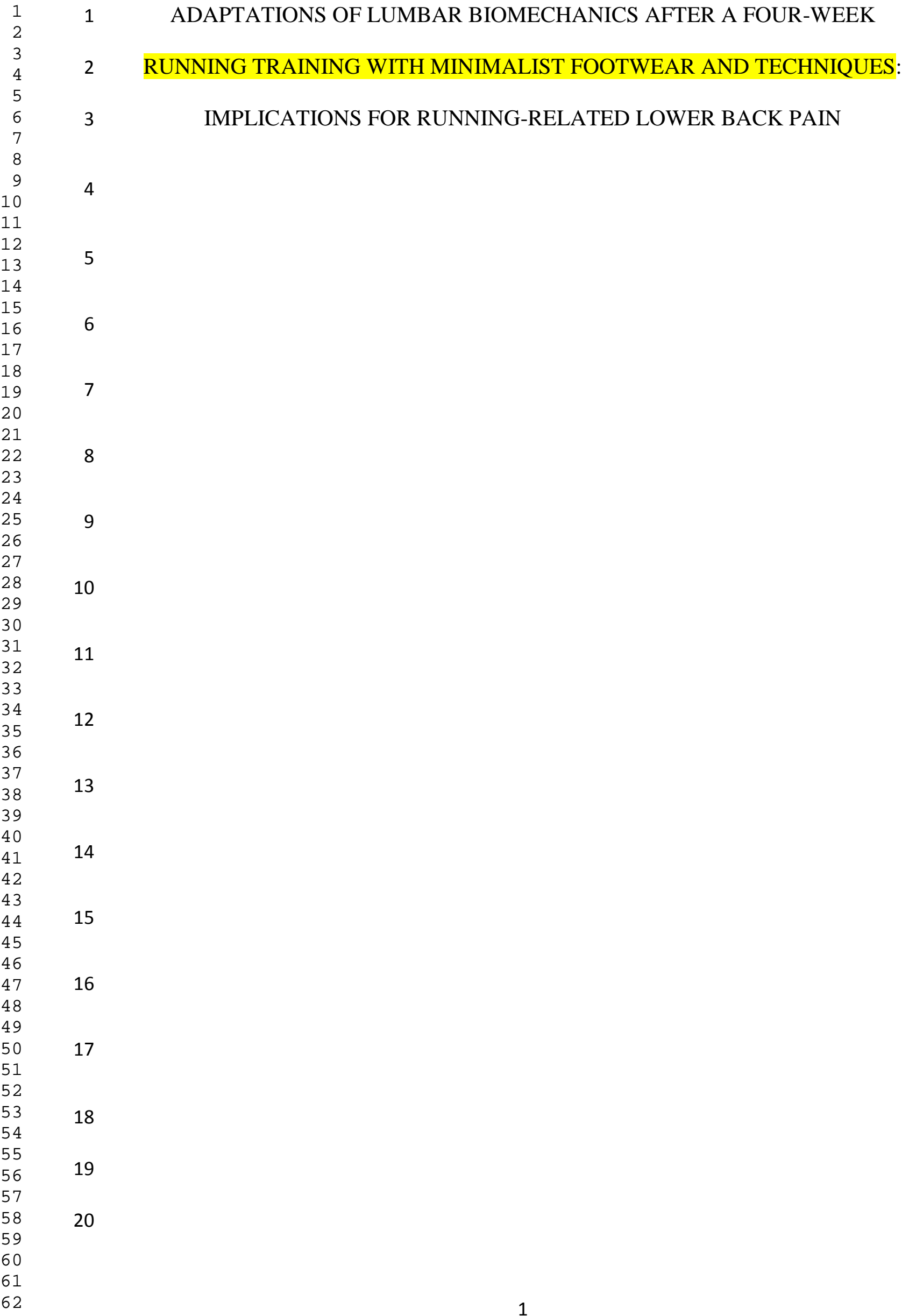




\section{$\underline{\text { ABSTRACT }}$}

22 Objectives: To investigate the changes in lumbar kinematic and paraspinal muscle

23 activation before, during, and after a 4-week minimalist running training.

24 Design: Prospective cohort study.

25 Setting: University research laboratory.

26 Participants: 17 habitually shod recreational runners who run 10-50 km per week.

27 Main outcome measures: During stance phases of running, sagittal lumbar kinematics

28 was recorded using an electro-goniometer and activities of the lumbar paraspinal

29 muscles were assessed with electromyography. Runners were asked to run at a

30 prescribed speed $(3.1 \mathrm{~m} / \mathrm{s})$ and a self-selected speed.

31 Results: For the $3.1 \mathrm{~m} / \mathrm{s}$ running speed, significant differences were found in the

32 calculated mean lumbar posture $(\mathrm{p}=0.001)$ during stance phase, specifically the runners

33 ran with a more extended lumbar posture after minimalist running training. A

34 significant reduction of the contralateral lumbar paraspinal muscle activation was also

35 observed $(\mathrm{p}=0.039)$. For the preferred running speed, similar findings of a more

36 extended lumbar posture $(\mathrm{p}=0.002)$ and a reduction in contralateral lumbar paraspinal

37 muscle activation $(\mathrm{p}=0.047)$ were observed.

38 Conclusion: A 4-week minimalist running training produced significant changes in

39 lumbar biomechanics during running. Specifically, runners adopted a more extended

40 lumbar posture and reduced lumbar paraspinal muscle activation. These findings may

41 have clinical implications for treating individuals with running-related lower back pain.

42 Key words: running; lower back pain; kinematics; EMG 
Running is one of the fastest expanding participation segments of sports and exercise. In the United States, It was estimated that 19 million people ran more than 100 times in 2011, a 9.3\% increase from 2010.(NSGA, 2011) The number of marathon

47 finishers increased by more than $75.5 \%$ in the last decade.(Lamppa, 2014) However, 48 the drastic increases in the popularity of running are accompanied by an increase in the 49 number of injured runners. Nielsen et al. reported that over the course of one year, $5023.1 \%$ of novice runners sustained running-related injuries to the lower extremity or back.(Nielsen, et al., 2014) According to a 2013 survey of running event participants, $10.1 \%$ of the runners reported experiencing a running-related lower back injury within 53 the last 12 months.(Yoder, 2013) Walter et al. have shown injuries pertaining to the 54 back and pelvis account for approximately $25-35 \%$ of all running-related injuries.(Walter, Hart, McIntosh, \& Sutton, 1989) In addition, preliminary data showed that running more than 20 miles per week can increase the odds of persistent LBP fivefold.(Gonzalez, Akuthota, Min, \& Sullivan, 2006)

The repetitive impact loading during running is a possible mechanism for developing lower back structural changes and pain in runners.(Cavanagh \& Lafortune, 1980; Dimitriadis, et al., 2011; Hamill, Gruber, \& Derrick, 2014; Hamill, Moses, \&

61 Seay, 2009) Dimitriadis et al. reported transient disc height reduction following 1 hour 62 of running measured using MRI in a static posture. Furthermore, the disc height 63 reduction was greatest in the lumbosacral region identifying a location of higher load 64 absorption.(Dimitriadis, et al., 2011) Garbutt et al. also observed that running speed is 65 positively related to the extent of stature shrinkage measured immediately after 66 running.(Garbutt, Boocock, Reilly, \& Troup, 1990) While acute structural changes of 
67 the spine are not directly indicative of pain, over time the mechanical stress associated with running's repetitive loading can potentially lead to changes in spinal structure and possibly overuse musculoskeletal symptoms including running-related lower back pain.

The recent interest in the body's natural ability to attenuate impact loads during running has led to a resurgence of barefoot and minimalist style running as a means to reduce the risk of running-related injuries.(Perkins, Hanney, \& Rothschild, 2014; Rixe,

73 Gallo, \& Silvis, 2012; Tam, Astephen Wilson, Noakes, \& Tucker, 2014) This running style typically focuses on running barefoot or wearing shoes with minimal heel cushion. Due to the reduced impact attenuation of the footwear, the runners typically adapt a change of foot strike pattern from rear to mid or forefoot and a reduction of peak impact force. In essence, the proposed benefits from running with the minimalist footwear were based on the theory that it promotes a movement pattern that is conducive to lower shock loading during running.(De Wit, De Clercq, \& Aerts, 2000;

It has been postulated that the biomechanical adaptations (i.e. foot strike pattern) associated with running barefoot or in minimalist footwear can lead to kinematic changes in the lumbo-pelvic region. For example, Delgado et al. reported decreased overall lumbar range of motion and peak leg impact measured via leg acceleration following an acute foot strike pattern shift from the rearfoot to

87 forefoot.(Delgado, et al., 2013) However, this study had a number of important

88 limitations: first, the effects of foot strike pattern on lumbar range of motions were

89 examined in a single data collection session. The participants were acutely instructed to 90 run using specific foot strike patterns, which may or may not translate to a more 
91 permanent movement pattern change. Second, the effect of minimalist running on

92 paraspinal muscle activation was not examined. Excessive paraspinal muscle activation

93 is hypothesized to contribute to increased lumbar spinal loading. Third, in practice, it 94 may be unrealistic and ill-advised to suggest drastic changes in foot strike and running 95 mechanics to injured or at-risk runners. It is clinically more meaningful to understand 96 the progression of responses in lumbar biomechanics to minimalist style running over a

97 longer duration of training.

The purpose of this study was to investigate the effects of a 4-week running training transitioning runners to minimalist footwear and techniques on the lumbar

100 kinematics and paraspinal muscle activation in habitually shod runners. We 101 hypothesized that the runners will exhibit a reduction of lumbar range of motion and 102 paraspinal muscle activation during the stance phase of running after training.

103 Methods

$104 \quad$ Subjects

105 Seventeen volunteers from the local running population was recruited. This 106 sample size was determined a priori based on a previous investigation on how change

107 of foot strike pattern affects lumbar posture.(Delgado, et al., 2013) To achieve an $80 \%$ 108 power, with $\alpha$ level of 0.05 , we calculated a sample size of 13 is needed to detect a 109 difference in a repeated measures study design. Additional 4 subjects were recruited to 110 account for potential attrition. The participants were included if they were: 1) age 18-45 111 years (Kienbacher, et al., 2015), 2) current recreational runners who run between 10-50

112 km during a typical week, and 3) habitual shod runners. Participants were excluded 113 from the study if they exhibited any of the following: previous experience with 
114 minimalist or barefoot running, any orthopedic surgeries that permanently change the

115 musculoskeletal structure of the lower extremity and spine (i.e. joint replacement,

116 spinal surgery, etc.), any injuries or conditions within the last 8 weeks that prevented

117 their normal running training. Two participants dropped out of the study due to an

118 unrelated injury and a personal reason, resulting in 8 male and 7 female participants

119 who completed the 4-week training program (Table 1).

120 TABLE 1. Demographic, anthropometric and running characteristics of the participants

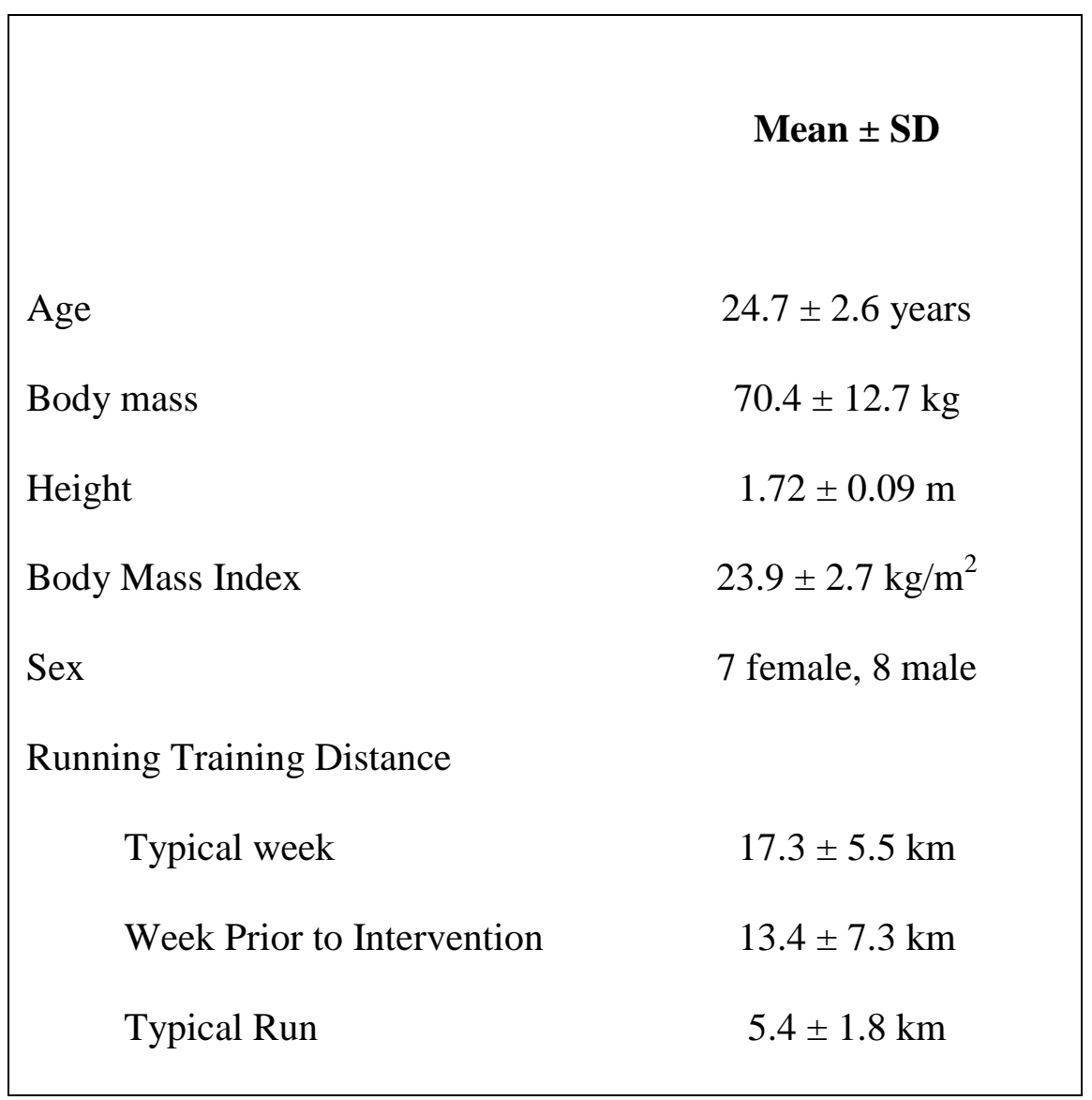

Prior to participation, the objectives, procedures, risks of the study, and rights of 123 the participant were explained to each participant, and an informed consent approved by the Institution Review Board of XXX University was signed by each participant. 
All testing was done with the participants running on a treadmill (PrecorC956;

127 Woodinville, WA, USA). Lumbar sagittal range of motion was captured using an 128 electrogoniometer (SG150/B Series; Biometrics Ltd., Newport, UK) connected to a 129 wireless transceiver (Delsys Trigno Biaxial Goniometer Adapter; Delsys Inc., Natick, 130 MA, USA). The center of the electrogoniometer was positioned over the spinous 131 processes of the $3^{\text {rd }}$ lumbar vertebrae. Sagittal plane lumbar range of motion was captured at $2000 \mathrm{~Hz}$. Electromyography (EMG) signals of the paraspinal muscles were captured using a wireless surface EMG system (Trigno Wireless System; Delsys Inc.,

134 Natick, MA, USA). Sampling frequency of the EMG signal was $2000 \mathrm{~Hz}$.

EMG preparation consisted of shaving the location to remove any hair,

136 cleansing the site with an alcohol swab, and abrading the site with a rough, dry paper 137 towel until the skin becomes flush in color. The EMG sensors were attached using 138 double-side tape. Pairs of surface EMG sensors were placed bilaterally over the 139 palpated lumbar paraspinal muscle bellies approximately $2-5 \mathrm{~cm}$ from the spinous 140 process of the $3^{\text {rd }}$ lumbar vertebrae. The electrodes were placed in parallel with the 141 fiber direction of the lumbar paraspinal muscle in accordance to the established surface 142 EMG protocol (Merletti, Rau, Disselhorst-Klug, Stegeman, \& Hagg, 2016; Zipp, 1982)

143 Foot strike incidents were monitored using 2 thin film pressure sensors (Model 144 402; Interlink Electronics Inc. Camarillo, CA, USA) placed inside of the shoes 145 connected through a Delsys wireless transceiver (Delsys Trigno 4-Channel FSR 146 Sensor). The sensors were attached to the bottom of the foot. The pressure sensor was 147 round and $12.7 \mathrm{~mm}$ in diameter with a thickness of $0.45 \mathrm{~mm}$. Foot pressure data was 
148 sampled at $2000 \mathrm{~Hz}$ for the rearfoot and $148 \mathrm{~Hz}$ for the forefoot. The difference in

149 sampling frequency was due to a hardware limitation. Since the duration of a stance

150 phase when running at the speeds used in this study $(2.8-3.5 \mathrm{~m} / \mathrm{s})$ was observed to be

$151350-500 \mathrm{~ms}$, we determined that the $148 \mathrm{~Hz}$ sampling frequency for the forefoot

152 pressure sensor would still provide sufficient temporal resolution $(6.76 \mathrm{~ms})$ to identify

153 the instant of toe-off with high degree of accuracy. EMG, lumbar kinematics, and foot

154 strike pressure data were time-synchronized through a trigger module (Delsys Trigger

155 Module; Delsys Inc., Natick, MA) to a motion capturing computer (Nexus 1.8, Vicon

156 Motion Systems Ltd., Oxford, UK).

\section{$157 \quad$ Procedure}

158 Each participant was tested in 3 sessions (PRE, MID, POST); the PRE session

159 was conducted on the day prior to the beginning of the 4-week training program; the

160 MID was at the 2-week point; the POST assessment was completed at the end of the

161 training (4-week). During each session, the testing began by measuring the runner's

162 height and weight, followed by instrumentation.

Maximal voluntary isometric contraction (MVIC) of back extension was 164 conducted with the subject in a prone position. The MVIC amplitude of the lumbar 165 paraspinal muscles was used to normalize the muscle activation level. The subject was 166 secured to a treatment table using straps. The tightness of the straps was adjusted to 167 elicit a neutral (lack of hyperextension) alignment during the back extension against the 168 strap. Two investigators provided additional stabilization of the legs as the participant performed two 5-second MVIC trials. 
170 Following the MVIC trials, the goniometer was attached to the participant in a

171 relaxed standing posture (Figure 1). The lumbar flexion/extension angle in this resting

172 standing position was defined as neutral $\left(0^{\circ}\right)$. The electrogoniometry procedure was

173 established in a prior study.(Delgado, et al., 2013) The two pressure switch sensors

174 were attached to the plantar surface of the foot of the dominant leg (defined as the 175 preferred leg to kick a ball with).

177 FIGURE 1. Placement of the EMG electrodes and the electrogoniometer

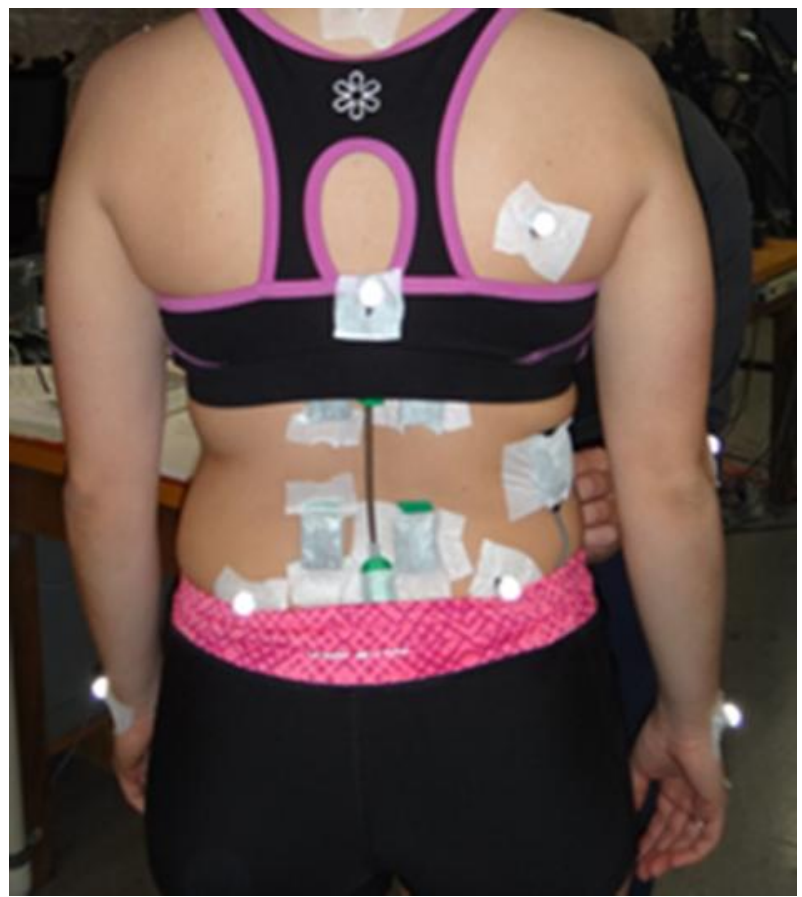

\section{Biomechanical Testing}

The testing began with a warm-up in which the participants walked on the

182 treadmill at $1.33 \mathrm{~m} / \mathrm{s}$ for 1 minute, the speed increased $0.22 \mathrm{~m} / \mathrm{s}$ at the end of every 
183 minute until the runner reached the prescribed running speeds. If the runner reported

184 discomfort during this period, the investigators stopped to make necessary adjustments

185 before the runner resumed the warm-up.

186 During all running trials, the participant wore his or her own running shoes. The

187 participant ran at two speeds: a control speed of $3.1 \mathrm{~m} / \mathrm{s}$ and a self-selected running

188 speed. For the preferred speed, the participant was blinded to the treadmill display and 189 an investigator changed the speed according to the runner's indication. Runners were 190 instructed to select a speed that felt close to their typical running training speed. Three 19120 -second trials were collected at each speed.

After the first running data collection session (PRE), participant was fitted with

a pair of standardized minimalist running shoes (Brooks ${ }^{\circledR}$ PureDrift; Brooks Sports,

\section{Minimalist Running Training Protocol}

The participants were instructed to begin by running $10 \%$ of their normal running mileage in the minimalist shoes. Every 2 weeks the participants would increase

200 the running distance wearing the minimalist shoes by no more than $10-20 \%$ of their 201 total running distance. This was intended to allow the runners to safely do $30-50 \%$ of 202 their running in the minimalist shoes by the end of week 4. This program was designed 203 based on the recommendation that minimalist shoe running should be gradually 204 incorporated into a person's normal running regimen to allow the body structures to 205 adapt to the different mechanical stressors.(Robillard, 2010, 2012) 
206 Each participant was given general instructions on minimalist running 207 techniques including maintaining relaxed shoulders, trunk, and a slight bend at the knee 208 throughout the running stride.(Robillard, 2010, 2012) The runners were also 209 recommended to try to land upon the forefoot as gently as possible. However, no 210 explicit feedback regarding each runner's running form was given during any of the 211 data collection sessions. This was done to simulate a common self-initiated transition to 212 the minimalist running shoes which is typically done with little external feedback or 213 guidance.

214 The runners were asked to keep a running log, including the time of each run, 215 the distance, and which shoes (normal or minimalist) they wore for the run. Each 216 participant was asked to record all of this information in their training log every day for 2174 weeks. The runners were asked not to change their normal training mileage. 218 Participants were advised to wear only their normal running shoes or the minimalist 219 shoes provided and not changing to different shoes during the 4-week 220 period. Participants were also instructed to perform a schedule of exercise drills 221 including: the Marble Drill, Jump Drill, and Walk in Place as typically suggested to 222 increase the strength of the feet (Table 2). 
228 TABLE 2. Weekly progression of the minimalist style running training

\begin{tabular}{|c|c|c|}
\hline & $\begin{array}{l}\text { Percentage mileage } \\
\text { recommended to run in } \\
\text { minimalist footwear }\end{array}$ & $\begin{array}{c}\text { Recommended exercise } \\
\text { drills to perform }\end{array}$ \\
\hline Week 1 & $10 \%$ & $\begin{array}{l}\text { Walk in Place } 2 x / \text { day } \\
\text { Marble drill 1x/day }\end{array}$ \\
\hline Week 2 & $20 \%$ & $\begin{array}{l}\text { Jump drill 2x/day } \\
\text { Walk in Place 2x/day } \\
\text { Marble drill 1x/day }\end{array}$ \\
\hline Week 3 & $20 \%-30 \%$ & $\begin{array}{l}\text { Jump drill 3x/day } \\
\text { Walk in Place 2x/day } \\
\text { Marble drill 1x/day }\end{array}$ \\
\hline Week 4 & $\geq 30 \%$ & $\begin{array}{l}\text { Jump drill 3x/day } \\
\text { Walk in Place 2x/day } \\
\text { Marble drill 1x/day }\end{array}$ \\
\hline
\end{tabular}

In the subsequent testing sessions (MID and POST), the weekly training logs

232 were reviewed. An exit questionnaire was given to each participant after the last testing

233 session (POST). The main question was whether they encounter any pain or injury 234 during the training.

\section{$235 \quad$ Data Analysis}

236 Changes in running distances wearing the normal and minimalist shoes over the

237 4-week training period were analyzed. The preferred running speeds in the two types of 238 shoes during the 3 testing sessions were recorded. Lumbar kinematics and muscle 239 activation data were computed during the stance phase of the dominant leg during the 240 running trials. The stance phases were identified with the aid of the foot pressure sensor 
241 data; specifically from the initial foot contact to when the forefoot lost contact with the

242 running surface.

$243 \quad$ For lumbar kinematics, mean sagittal lumbar posture, peak lumbar flexion, peak

244 lumbar extension, and lumbar range of motion were computed. The mean sagittal

245 lumbar posture was the time-averaged lumbar posture during the stance phase; a

246 positive angular value indicates lumbar flexion. Mean lumbar muscle activation during

247 the stance phase was computed for both the contralateral and the ipsilateral paraspinal

248 muscles. The EMG signals from the lumbar paraspinal muscles were first filtered with

249 a $2^{\text {nd }}$ order Butterworth band-pass filter (cut-off frequencies: 35-500 Hz) then full-wave

250 rectified. The mean muscle activation magnitudes were normalized to the highest 500

251 millisecond average activation magnitude obtained during the MVIC trials, and

252 reported as percentages of the MVIC. This duration was chosen to correspond with the

253 approximate duration of the stance phase $(350-500 \mathrm{~ms})$ for the running speeds used in

254 this study. For each running trial, 10 stance phases were identified; the lumbar

255 kinematic and muscle activation magnitude variables were obtained by averaging over

256 the 10 stance phases from each trial. The average values from 3 running trials for each

257 subject were used for statistical analysis.

\section{$258 \quad$ Statistical Analysis}

259 One-way repeated measures ANOVA tests were used to compare the 260 participants' preferred running speeds, lumbar kinematic, and muscle activation 261 variables during the 4-week training program (PRE, MID, and POST). Biomechanical 262 data obtained from the $3.1 \mathrm{~m} / \mathrm{s}$ and the preferred running speeds were analyzed 263 separately. Post-hoc comparisons with Bonferroni correction were conducted when the 
264 main effect was significant. All statistical procedures were conducted using SPSS® 26522.0 (International Business Machines Corp. New York, USA). Significance level was 266 set at 0.05 .

$\underline{\text { Results }}$

The reported weekly mileage per footwear condition during the 4-week protocol is presented in Table 3. The percentage of total running distance in the minimalist shoes

270 gradually increased from $18.8 \%$ to $54.9 \%$ during the 4 weeks. A significant difference 271 was detected in the preferred running speed $(\mathrm{p}=0.007)$ that at the MID the preferred 272 running speed was significantly slower than at PRE (PRE vs. MID, 3.25 \pm 0.33 vs. $2733.13 \pm 0.31 \mathrm{~m} / \mathrm{s}, \mathrm{p}=0.016)$. No other differences in running speed were detected.

TABLE 3. Recorded weekly distance ran by participants in minimalist and their 276 normal running shoes.

\begin{tabular}{|c|c|c|c|c|}
\hline Running Distance (km) & Week 1 & Week 2 & Week 3 & Week 4 \\
\hline Total in minimalist shoes & $3.6 \pm 3.4$ & $4.2 \pm 2.2$ & $7.1 \pm 2.9$ & $7.8 \pm 4.0$ \\
\hline Average per run in minimalist shoes & $2.6 \pm 2.0$ & $3.8 \pm 2.0$ & $4.3 \pm 1.4$ & $4.7 \pm 1.5$ \\
\hline $\begin{array}{c}\text { Total in normal shoes } \\
\text { Average per run in normal shoes }\end{array}$ & $14.5 \pm 7.4$ & $12.5 \pm 6.4$ & $9.8 \pm 6.3$ & $6.4 \pm 3.8$ \\
\hline $\begin{array}{c}\text { \% of total distance in minimalist } \\
\text { shoes }\end{array}$ & $18.8 \%$ & $31.3 \%$ & $42.1 \%$ & $54.9 \%$ \\
\hline \multicolumn{2}{c}{ sho } & $5.4 \pm 2.1$ & $5.8 \pm 4.8$ & $5.1 \pm 3.6$ \\
\hline
\end{tabular}

277 
278 During the prescribed $3.1 \mathrm{~m} / \mathrm{s}$ running speed, significant differences were 279 detected in mean lumbar posture, peak flexion, peak extension, and contralateral 280 paraspinal muscle activation between the 3 testing sessions (Table 4). Post-hoc 281 comparisons showed that the mean lumbar posture was significantly less flexed when 282 compared to before training (PRE vs. POST, $1.9 \pm 15.3^{\circ}$ vs. $-6.0 \pm 13.3^{\circ}, \mathrm{p}=0.001$ ). Peak 283 lumbar flexion angle was significantly lower after training (PRE vs. POST, 8.6 $\pm 15.7^{\circ}$ 284 vs. $-0.3 \pm 13.7^{\circ}, \mathrm{p}<0.001$; MID vs. POST, $7.6 \pm 15.1^{\circ}$ vs. $\left.-0.3 \pm 13.7^{\circ}, \mathrm{p}=0.001\right)$. Peak 285 lumbar extension angle increased significantly after training (PRE vs. POST, $4.8 \pm 14.3^{\circ}$ 286 vs. $6.7 \pm 11.8^{\circ}, \mathrm{p}<0.001 ; \mathrm{MID}$ vs. $\mathrm{POST}, 6.7 \pm 11.8^{\circ}$ vs. $\left.12.6 \pm 12.4^{\circ}, \mathrm{p}=0.033\right)$. There was 287 no significant change in the overall lumbar range of motion before, during, and after 288 training. The contralateral lumbar paraspinal muscle activation significantly differed 289 among the 3 time points. Post-hoc comparison showed that there was a significant 290 reduction of muscle activation after two weeks of training (PRE vs. MID, 47.0 $\pm 34.0 \%$ 291 vs. $24.9 \pm 8.2 \%, \mathrm{p}=0.049)$. No significant difference in muscle activation was observed 292 in the ipsilateral paraspinal muscle. 
299 TABLE 4. Comparison of lumbar kinematic and paraspinal muscle activation pre, mid, 300 and post the 4-week training.

\begin{tabular}{|c|c|c|c|c|c|c|c|c|}
\hline & \multicolumn{4}{|c|}{$3.1 \mathrm{~m} / \mathrm{s}$ running speed } & \multicolumn{4}{|c|}{ Preferred running speed } \\
\hline & PRE & MID & POST & $\begin{array}{c}p \\
\text { value }\end{array}$ & PRE & MID & POST & $\begin{array}{c}p \\
\text { value }\end{array}$ \\
\hline $\begin{array}{l}\text { Mean lumbar } \\
\text { posture } \\
\text { (degree) }\end{array}$ & $\begin{array}{c}1.9 \pm \\
15.3\end{array}$ & $\begin{array}{c}0.4 \pm \\
13.0\end{array}$ & $\begin{array}{c}-6.0 \pm \\
13.3^{*}\end{array}$ & 0.001 & $\begin{array}{c}2.3 \pm \\
15.5\end{array}$ & $\begin{array}{c}0.9 \pm \\
13.9\end{array}$ & $\begin{array}{l}-5.7 \pm \\
14.2^{*}\end{array}$ & 0.002 \\
\hline $\begin{array}{l}\text { Peak lumbar } \\
\text { flexion } \\
\text { (degree) }\end{array}$ & $\begin{array}{c}8.6 \pm \\
15.7\end{array}$ & $\begin{array}{c}7.6 \pm \\
15.1\end{array}$ & $\begin{array}{l}-0.3 \pm \\
13.7 * \dagger\end{array}$ & $<0.001$ & $\begin{array}{c}9.1 \pm \\
16.3\end{array}$ & $\begin{array}{c}8.0 \pm \\
15.4\end{array}$ & $\begin{array}{l}-0.3 \pm \\
14.7 *^{\dagger}\end{array}$ & $<0.001$ \\
\hline $\begin{array}{l}\text { Peak lumbar } \\
\text { extension } \\
\text { (degree) }\end{array}$ & $\begin{array}{c}4.8 \pm \\
14.3\end{array}$ & $\begin{array}{c}6.7 \pm \\
11.8\end{array}$ & $\begin{array}{l}12.6 \pm \\
12.4 *^{\dagger}\end{array}$ & 0.005 & $\begin{array}{c}4.4 \pm \\
14.7\end{array}$ & $\begin{array}{c}6.7 \pm \\
12.5\end{array}$ & $\begin{array}{l}12.4 \pm \\
13.5^{* \dagger}\end{array}$ & 0.007 \\
\hline $\begin{array}{l}\text { Overall } \\
\text { lumbar ROM } \\
\text { (degree) }\end{array}$ & $\begin{array}{r}13.3 \\
\pm 2.4\end{array}$ & $\begin{array}{r}14.3 \\
\pm 6.1\end{array}$ & $\begin{array}{c}12.3 \pm \\
4.4\end{array}$ & 0.496 & $\begin{array}{r}13.5 \\
\pm 2.4\end{array}$ & $\begin{array}{c}14.7 \pm \\
6.0\end{array}$ & $\begin{array}{c}12.1 \pm \\
4.6\end{array}$ & 0.325 \\
\hline $\begin{array}{l}\text { Contralateral } \\
\text { lumbar muscle } \\
\text { activation } \\
(\% \text { of MVIC) }\end{array}$ & $\begin{array}{c}47.0 \\
\pm \\
34.0\end{array}$ & $\begin{array}{c}24.9 \\
\pm \\
8.2^{*}\end{array}$ & $\begin{array}{c}29.4 \pm \\
11.3\end{array}$ & 0.039 & $\begin{array}{c}41.6 \\
\pm \\
28.6\end{array}$ & $\begin{array}{c}23.4 \pm \\
6.2\end{array}$ & $\begin{array}{c}30.3 \pm \\
11.6\end{array}$ & 0.047 \\
\hline $\begin{array}{l}\text { Ipsilateral } \\
\text { lumbar muscle } \\
\text { activation } \\
\text { (\% of MVIC) }\end{array}$ & $\begin{array}{c}26.5 \\
\pm \\
15.8\end{array}$ & $\begin{array}{r}17.0 \\
\pm 4.1\end{array}$ & $\begin{array}{c}25.5 \pm \\
17.2\end{array}$ & 0.225 & $\begin{array}{c}28.8 \\
\pm \\
22.5\end{array}$ & $\begin{array}{c}16.7 \pm \\
3.8\end{array}$ & $\begin{array}{c}25.9 \pm \\
17.8\end{array}$ & 0.262 \\
\hline
\end{tabular}

*indicates a significant difference from PRE condition.

$302 \uparrow$ indicates a significant diffidence from the MID condition.

For the preferred running speed, significant differences were detected in mean 305 lumbar posture, peak lumbar flexion, peak lumbar extension, and contralateral 306 paraspinal lumbar muscle activation between the 3 testing sessions (Table 4). Post-hoc comparisons showed that the mean lumbar posture was significantly less flexed when compared to before training (PRE vs. POST, $2.3 \pm 15.5^{\circ}$ vs. $-5.7 \pm 14.2^{\circ}, \mathrm{p}=0.002$ ). Peak 
310 vs. $\left.-0.3 \pm 14.7^{\circ}, \mathrm{p}<0.001\right)$. The peak lumbar extension angle increased significantly after training (PRE vs. POST, $4.4 \pm 14.7^{\circ}$ vs. $12.4 \pm 13.5^{\circ}, \mathrm{p}<0.001$; MID vs. POST, $6.7 \pm 12.5^{\circ}$

312 vs. $\left.12.4 \pm 13.5^{\circ}, \mathrm{p}=0.046\right)$. There was no significant change in the overall lumbar range 313 of motion before, during, and after the training. The contralateral lumbar paraspinal 314 muscle activation significantly differed among the 3 time points. The post-hoc comparison detected a trend of reduction in contralateral paraspinal muscle activation after two weeks of training (PRE vs. MID, $41.6 \pm 28.6 \%$ vs. $23.4 \pm 6.2 \%, \mathrm{p}=0.072$ ). No significant difference in muscle activation was observed in the ipsilateral paraspinal muscle.

\section{$\underline{\text { Discussion }}$}

Biomechanical evaluations aimed at identifying risk factors, prevention, and treatment strategies pertinent to running-related injuries have traditionally focused on the more common injuries such as knee pain and tendinopathy. In comparison, research regarding the biomechanics of lumbar spine during running is lacking. This is an important void in the current knowledge base that needs to be addressed, because lower back dysfunctions are relatively common in distance runners.(Gonzalez, et al., 2006; Walter, et al., 1989; Yoder, 2013) Also, preliminary evidence suggested that dysfunction or weakness of the lumbar-pelvis-hip musculoskeletal complex can lead to injuries in other parts of the body.(Brumitt, 2011; Hammill, Beazell, \& Hart, 2008)

Our primary finding was that the runners gradually adopted a more extended lumbar posture over the 4 weeks of training. During running, lumbar flexion has been shown to dominate the initial loading phase of stance followed by more extension from midstance to toe-off.(Saunders, Schache, Rath, \& Hodges, 2005; Schache, Blanch, Rath, Wrigley, \& Bennell, 2002; Schache, Blanch, Rath, Wrigley, \& Bennell, 2005; 
334 Schache, et al., 2001) Lumbar flexion during the initial loading phase is thought to aid 335 in the attenuation of the impact forces.

336 In order to provide effective intervention to runners with running-related lower 337 back pain, it is important to establish how different running styles may affect spinal 338 mechanics. Changes in lumbar kinematics in response to foot strike pattern during 339 running were first reported by Delgado et al.(Delgado, et al., 2013) The authors 340 reported a small reduction (from 22.1 to $20.9^{\circ}$ ) in overall lumbar range of motion after 341 acute changes of foot strike pattern. Contrary to their findings, we observed no changes 342 in the lumbar range of motion, but an overall tendency of most runners adopting a more 343 extended lumbar posture after running training with minimalist footwear and technique 344 instruction. Specifically, the runners in our study generally exhibited a gradual 345 reduction in peak lumbar flexion angle over the course of the training (Figure 2). The 346 discrepancy in the results perhaps stemmed from the different methodology. In the 347 previous study the peak lumbar spinal angles were recorded over combined swing and 348 stance phases, while in this study we focused on the stance phase. Also, we did not 349 explicitly instruct the runners on the foot strike location during the running trials, but 350 allowed the runners to naturally adapt to running with the minimalist footwear over 351 time. 
358 FIGURE 2 . Changes of mean lumbar posture during the stance phase of running for 359 each participant Pre, Mid, and Post the 4-week training

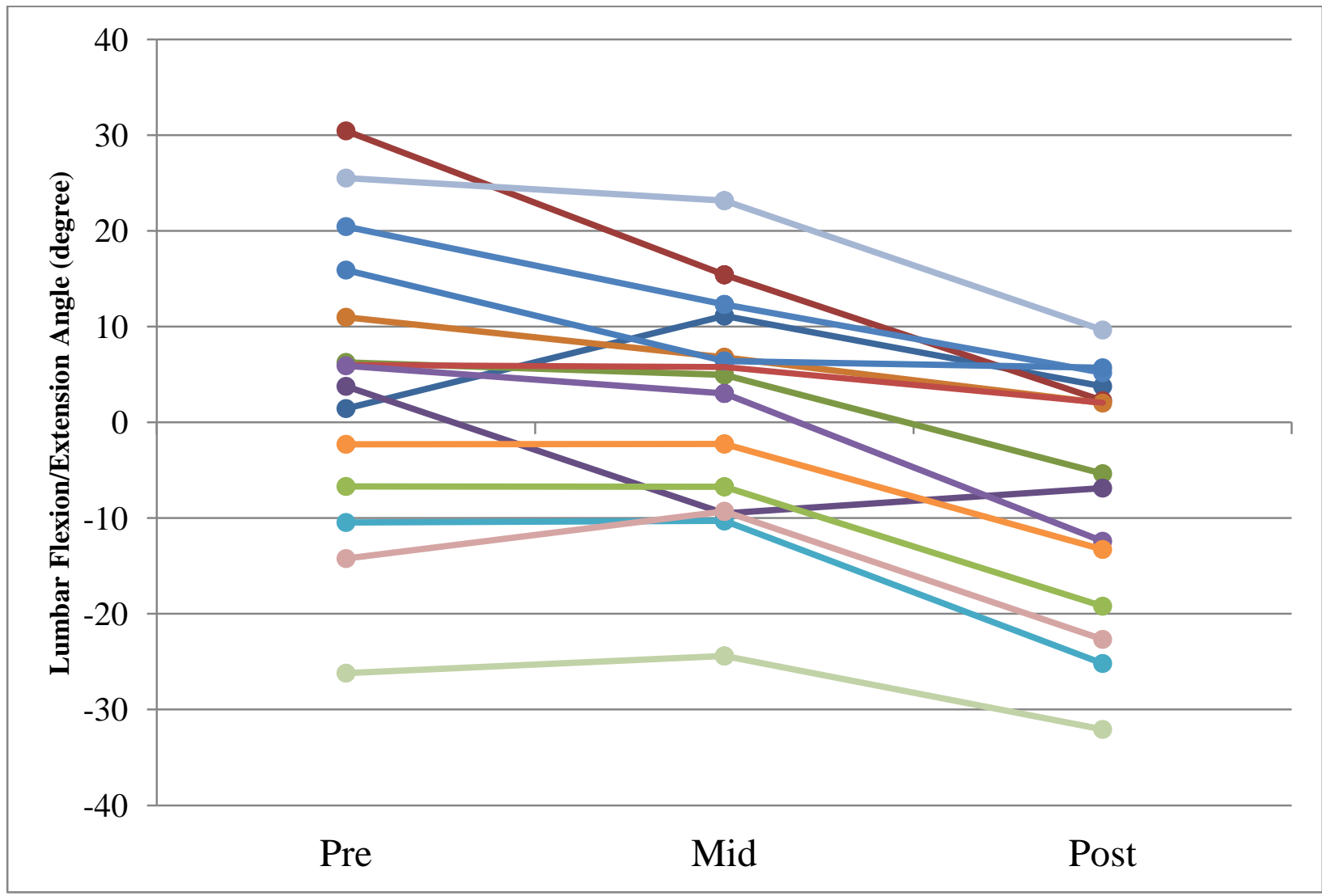

The observed more upright running posture after training was accompanied by

363 reduced contralateral paraspinal muscle activation. Previous studies have shown that 364 the greatest activation of the lumbar paraspinal muscle group occurs during forward 365 trunk flexion and is reduced during extension.(Kienbacher, et al., 2015) The observed 366 reduction in the contralateral muscle activation during stance is compatible with the 367 reduced need to stabilize the lumbar spine against gravity in the more upright posture 368 and the potential reduction of impact shock after training.

Appropriate muscle activation during running facilitates adequate coordination

370 between the lumbar spine, pelvis and hip complex, helps to stabilize the spine in 
371 response to the ground reaction force during the stance phase of running. However,

372 excessive lumbar paraspinal muscle activation may be a sign of dysfunction, and the

373 increased muscular force can lead to increased loading on the spine. Previous EMG

374 studies of lumbar muscle activation demonstrated that during locomotion, patients with

375 LBP showed continuous activation in contrast to the more phasic activation pattern

376 observed in those without LBP.(Kuriyama \& Ito, 2005; van der Hulst, et al., 2010) This

377 indicates that patients with LBP exhibit altered paraspinal muscle activation patterns,

378 perhaps as a guarding response that also increases the stiffness of the spine. Such

379 increased loading from the paraspinal muscle contraction may also be related to the

380 chronic back pain symptoms and interferes with recovery. While we could not

381 definitively imply the observed reduction of paraspinal muscle activation as beneficial,

382 it is possible that such change can be clinically meaningful. Future intervention study

383 on runners with running-related lower back pain is needed to investigate the clinical

384 utility of minimalist running for treating this condition.

385 Fifteen out of 17 runners were able to complete the training, and none of them 386 reported any running-related injuries during the training period. Our 4-week training

387 intervention protocol was designed around a gradual progression of runners' weekly 388 training mileage in the minimalist shoes. Some previous study protocols were longer at 389 10-12 weeks.(Miller, Whitcome, Lieberman, Norton, \& Dyer, 2014; Ridge, et al., 390 2013) The extended durations in those studies were necessary for identifying 391 musculoskeletal structural adaptations to the adjusted stress from the altered running 392 pattern. On the other hand, the focus of the current study was to identify movement 393 pattern adjustments rather than structural adaptations. Furthermore, the end point of our 
394 program was to allow the runners to incorporate minimalist footwear running into only $30-50 \%$ of their total weekly mileage and not a full conversion.

In the current study, the minimalist running training was designed as a supplement to the runner's typical running routine with the inclusion of footwear variability, postural cues and strengthening exercises. In other words, the minimalist style running was used as an exercise drill to induce changes in running movement pattern, which we observed to be transferrable to the runners' normal shod running. This could imply that some learning occurred due to the running training used in this study. We believe that this finding is clinically important as it is often unrealistic to ask a runner to completely shift to a different running style or footwear. In fact, results from a number of previous studies have shown that even successful complete transition to minimalist running can induce potential damage to the foot and lower extremity.(Ridge, et al., 2013; Ryan, Elashi, Newsham-West, \& Taunton, 2014) While researchers and clinicians continue to debate about the benefits and injury risks associated with minimalist running,(Jenkins \& Cauthon, 2011; Perkins, et al., 2014) it is likely safer to utilize the minimalist style running as a supplemental training to induce beneficial movement pattern changes and not to emphasize a complete transition.

This study has a number of limitations. The biomechanical testing was done on a treadmill, which may not reflect the activities of the lumbar spine and paraspinal muscle during overground running as the treadmill afforded some cushioning. Also, direct measurement of ground reaction force was not done. Since foot strike impact attenuation has been proposed as an important factor to running-related lower back dysfunctions,(Hamill, et al., 2009) future studies should examined the ground reaction 
418 impact attenuation and energy absorption of the lower extremity and spinal joints.

419 While surface EMG provide a reliable method of quantifying trunk muscle activity

420 during running locomotion,(Smoliga, Myers, Redfern, \& Lephart, 2010) they are

421 unable to differentiate between activity in the different depth of muscles that comprise

422 the lumbar paraspinal group.(Stokes, Henry, \& Single, 2003) Lastly, it is important to

423 recognize that individual response to training differs. A recent study has shown that

424 only certain runners respond to barefoot running training in a manner consistent with

425 injury prevention.(Tam, Tucker, \& Astephen Wilson, 2016) Future research should

426 focus on the feasibility and the clinical benefits of minimalist style running in clinical

427 populations.

\section{$428 \quad$ Conclusion}

Our results demonstrated that a 4-week running training with minimalist

430 footwear and techniques instruction can induce significance changes to lumbar spine

431 biomechanics during running. Specifically, the participants ran with a less flexed, and

432 more upright lumbar posture after training. Correspondingly, we observed a trend of

433 reduction of the contralateral lumbar paraspinal muscle activation. These effects were

434 observed when the runners ran wearing their regular running footwear. Our findings

435 demonstrated that including minimalist style running into a runners' training may

436 induce beneficial changes to the lumbar kinematics and paraspinal muscle activation

437 during their normal shod running. 


\section{REFERENCES}

441 Brumitt, J. (2011). Successful rehabilitation of a recreational endurance runner: initial 442 validation for the Bunkie test. J Bodyw Mov Ther, 15, 384-390.

443 Cavanagh, P. R., \& Lafortune, M. A. (1980). Ground reaction forces in distance running. $J$ $444 \quad$ Biomech, 13, 397-406.

445 De Wit, B., De Clercq, D., \& Aerts, P. (2000). Biomechanical analysis of the stance phase 446 during barefoot and shod running. J Biomech, 33, 269-278.

447 Delgado, T. L., Kubera-Shelton, E., Robb, R. R., Hickman, R., Wallmann, H. W., \& Dufek, J. 448 S. (2013). Effects of foot strike on low back posture, shock attenuation, and comfort in $449 \quad$ running. Med Sci Sports Exerc, 45, 490-496.

450 Derrick, T. R., \& Mercer, J. A. (2004). Ground/foot impacts: measurement, attenuation, and $451 \quad$ consequences. Med Sci Sports Exerc, 36, 830-831.

452 Dimitriadis, A. T., Papagelopoulos, P. J., Smith, F. W., Mavrogenis, A. F., Pope, M. H., 453 Karantanas, A. H., Hadjipavlou, A. G., \& Katonis, P. G. (2011). Intervertebral disc $454 \quad$ changes after $1 \mathrm{~h}$ of running: a study on athletes. J Int Med Res, 39, 569-579.

455 Divert, C., Mornieux, G., Baur, H., Mayer, F., \& Belli, A. (2005). Mechanical comparison of 456 barefoot and shod running. Int J Sports Med, 26, 593-598.

457 Garbutt, G., Boocock, M. G., Reilly, T., \& Troup, J. D. (1990). Running speed and spinal 458 shrinkage in runners with and without low back pain. Med Sci Sports Exerc, 22, 769459 460 Gonzalez, P., Akuthota, V., Min, S. J., \& Sullivan, W. J. (2006). The Prevalence of Low Back 461 Pain in Recreational Distance Runners. Med Sci Sports Exerc, 38, S349-S349.

462 Hamill, J., Gruber, A. H., \& Derrick, T. R. (2014). Lower extremity joint stiffness 463 characteristics during running with different footfall patterns. Eur J Sport Sci, 14, 130464 136. 
465 Hamill, J., Moses, M., \& Seay, J. (2009). Lower extremity joint stiffness in runners with low 466 back pain. Res Sports Med, 17, 260-273.

Hammill, R. R., Beazell, J. R., \& Hart, J. M. (2008). Neuromuscular consequences of low back pain and core dysfunction. Clin Sports Med, 27, 449-462, ix.

Jenkins, D. W., \& Cauthon, D. J. (2011). Barefoot running claims and controversies: a review of the literature. J Am Podiatr Med Assoc, 101, 231-246.

Kienbacher, T., Paul, B., Habenicht, R., Starek, C., Wolf, M., Kollmitzer, J., Mair, P., \& Ebenbichler, G. (2015). Age and gender related neuromuscular changes in trunk flexion-extension. J Neuroeng Rehabil, 12, 3 .

Kuriyama, N., \& Ito, H. (2005). Electromyographic functional analysis of the lumbar spinal muscles with low back pain. J Nippon Med Sch, 72, 165-173.

Lamppa, R. (2014). Running USA Annual Marathon Report. In.

Mercer, J. A., Vance, J., Hreljac, A., \& Hamill, J. (2002). Relationship between shock attenuation and stride length during running at different velocities. Eur J Appl Physiol, $87,403-408$.

Merletti, R., Rau, G., Disselhorst-Klug, C., Stegeman, i. D. F., \& Hagg, G. M. (2016). Surface Electromyography for the Non-Invasive Assessment of Muscles (SENIAM). In: Biomedical Health and Research Program (BIOMED II) of the European Union.

Miller, E. E., Whitcome, K. K., Lieberman, D. E., Norton, H. L., \& Dyer, R. E. (2014). The effect of minimal shoes on arch structure and intrinsic foot muscle strength. $J$ Sport Health Sci, 3, 74-85.

Nielsen, R. O., Parner, E. T., Nohr, E. A., Sorensen, H., Lind, M., \& Rasmussen, S. (2014). Excessive progression in weekly running distance and risk of running-related injuries: an association which varies according to type of injury. J Orthop Sports Phys Ther, 44, 739-747. 
NSGA. (2011). National Sporting Goods Association: Sports Participation - Ranked by Total Participation. In.

Perkins, K. P., Hanney, W. J., \& Rothschild, C. E. (2014). The risks and benefits of running barefoot or in minimalist shoes: a systematic review. Sports Health, 6, 475-480.

Ridge, S. T., Johnson, A. W., Mitchell, U. H., Hunter, I., Robinson, E., Rich, B. S., \& Brown, S. D. (2013). Foot bone marrow edema after a 10-wk transition to minimalist running shoes. Med Sci Sports Exerc, 45, 1363-1368.

Rixe, J. A., Gallo, R. A., \& Silvis, M. L. (2012). The barefoot debate: can minimalist shoes reduce running-related injuries? Curr Sports Med Rep, 11, 160-165.

Robbins, S. E., \& Hanna, A. M. (1987). Running-related injury prevention through barefoot adaptations. Med Sci Sports Exerc, 19, 148-156.

Robillard, J. (2010). The barefoot running book: a practical guide to the art and science of barefoot \& minimalist shoe running. Michigan: Jason Robillard.

Robillard, J. (2012). The barefoot running book : the art and science of barefoot and minimalist shoe running. New York: Plume.

Ryan, M., Elashi, M., Newsham-West, R., \& Taunton, J. (2014). Examining injury risk and pain perception in runners using minimalist footwear. Br J Sports Med, 48, 1257-1262.

Saunders, S. W., Schache, A., Rath, D., \& Hodges, P. W. (2005). Changes in three dimensional lumbo-pelvic kinematics and trunk muscle activity with speed and mode of locomotion. Clin Biomech (Bristol, Avon), 20, 784-793.

Schache, A. G., Blanch, P., Rath, D., Wrigley, T., \& Bennell, K. (2002). Three-dimensional angular kinematics of the lumbar spine and pelvis during running. Hum Mov Sci, 21, 273-293.

Schache, A. G., Blanch, P. D., Rath, D. A., Wrigley, T. V., \& Bennell, K. L. (2005). Are anthropometric and kinematic parameters of the lumbo-pelvic-hip complex related to running injuries? Res Sports Med, 13, 127-147. 
516 Schache, A. G., Blanch, P. D., Rath, D. A., Wrigley, T. V., Starr, R., \& Bennell, K. L. (2001).

517

518

519 A comparison of overground and treadmill running for measuring the threedimensional kinematics of the lumbo-pelvic-hip complex. Clin Biomech (Bristol, Avon), 16, 667-680.

Smoliga, J. M., Myers, J. B., Redfern, M. S., \& Lephart, S. M. (2010). Reliability and precision of EMG in leg, torso, and arm muscles during running. J Electromyogr Kinesiol, 20, e1-9.

Stokes, I. A., Henry, S. M., \& Single, R. M. (2003). Surface EMG electrodes do not accurately record from lumbar multifidus muscles. Clin Biomech (Bristol, Avon), 18, 9-13.

Tam, N., Astephen Wilson, J. L., Noakes, T. D., \& Tucker, R. (2014). Barefoot running: an evaluation of current hypothesis, future research and clinical applications. Br J Sports Med, 48, 349-355.

Tam, N., Tucker, R., \& Astephen Wilson, J. L. (2016). Individual Responses to a Barefoot Running Program: Insight Into Risk of Injury. Am J Sports Med, 44, 777-784.

van der Hulst, M., Vollenbroek-Hutten, M. M., Rietman, J. S., Schaake, L., GroothuisOudshoorn, K. G., \& Hermens, H. J. (2010). Back muscle activation patterns in chronic low back pain during walking: a "guarding" hypothesis. Clin J Pain, 26, 30-37.

Walter, S. D., Hart, L. E., McIntosh, J. M., \& Sutton, J. R. (1989). The Ontario cohort study of running-related injuries. Arch Intern Med, 149, 2561-2564.

Yoder, T. (2013). 2013 National Runner Survey by Running USA. In.

Zipp, P. (1982). Recommendations for the Standardization of Lead Positions in Surface Electromyography. European Journal of Applied Physiology and Occupational Physiology, 50, 41-54. 


\section{${ }^{*}$ Acknowledgements}

\section{Acknowledgements}

This research was supported by the Faculty Opportunity Award from the Provost's Office and the Student Opportunity Research Grant from the Department of Physical Therapy, University of Nevada, Las Vegas. We also would like to acknowledge the support provided by the University of Nevada, Las Vegas Sports Injury Research Center. 